\title{
Mood Prediction Reflecting Emotion State to Improve Mental Health
}

\author{
Shinya Yonekura ${ }^{1}$, Yusuke Kajiwara ${ }^{2}$ and Hiromitsu Shimakawa ${ }^{2}$
}

\begin{abstract}
In this paper, we try to predict a mood using cognitive emotions. We investigate what kind of events affects the future mood. The mood prediction allows youths to grasp their depression in the future. We construct a mood prediction model with biological information, weather information, and cognitive emotion. The average of the prediction accuracy is $1.2 \%$ higher than that without them. We found emotion such as leisure affects the prediction accuracy.
\end{abstract}

Keywords - mood prediction, mental health, emotion, machine learning.

\section{INTRODUCTION}

The incidence of mental health afflictions has become a serious problem in the world. An investigation by the WHO (2014) indicated around $20 \%$ of children and adolescents in the world have mental disorders or various problems[1]. Generally speaking, self-care is effective to prevent mental disorders. However, many youths cannot take self-care because it is too difficult for them to grasp what their own mood will be in the future. Consequently, they believe they are healthy even when they feel stress. The accumulation of stress finally goes beyond their capacity. They fall into mental disorders such as depression.

It is necessary to help them grasp their future mood. Prediction of long-lasting mood in the future enables youths to prevent them from falling into mental disorder.

\section{RELATION BETWEEN MOOD AND EMOTION}

\section{A. Difference of Mood and Emotion}

In psychology, mood is clearly discriminated from emotion. As Eagle[2] and Frijida[3] mentioned, mood is feeling which needs a long time to change. It is hard to identify causes of its changes. On the contrary, Sloboda[4] said emotion changes rapidly. We can identify causes of its change. In addition, strong emotion affects mood as well as emotion changes bring mood changes[5]. In order to predict future mood, it is important to understand how emotion changes and to find main factors in emotion changes. Many studies have proposed definitions of what emotion is. As the James-Lange theory mentions, emotion is caused by physiological changes due to a stimulus. For example, when a person gets angry, the

\footnotetext{
${ }^{1}$ Graduate School of Information Science and Engineering Ritsumeikan University, Shiga, Japan

${ }^{2}$ College of Information Science and Engineering Ritsumeikan University, Shiga, Japan
}

blood pulse first raises, and then emotion appears. However, Canon[6] denies this theory. As Lazarus mentioned, emotion must have some cognitive intentionality[7]. Emotion is a disturbance that occurs in the following order.

1. Cognitive appraisal phase: the individual experience an event, which cues the emotion. The individual assesses cognitively the event.

2. Physiological change phase: the cognitive reaction starts biological change, such as increase in the blood pulse or sweating.

3. Action phase: the individual feels the emotion and chooses how to react.

Let us consider an example which occurs to Tom in the following scenario.

1. Tom gets a new task, and cognitively assesses the task. Cognition allows him to understand it is a too hard.

2. His brain activates the adrenal glands, which pump adrenaline through his blood stream, resulting in blood pulse increase.

3. Tom gets a cold sweat from impatience and gets cracking.

Lazarus insisted that the quality and intensity of emotions are controlled through cognitive processes. Based on Lazarus's theory, cognition such as expectation should affect future mood. This paper founds on Lazarus's theory.

\section{B. Related Study}

Katsimerou predicts the mood using punctual emotion appearing during video watching[8]. Katsimerou shows that exponential discount of the past emotions achieves the prediction accuracy of mood above $60 \%$. The problem seems to lie in the fact that it does not consider how to improve future mood. As Wu mentioned, it is important to predict changing emotion, using human respiration signal[9]. The average of the accuracy predicting five prototypical emotions (i.e., "love," "sadness," "joy," "anger," and "fear") is $88 \%$. However, it is difficult to extract emotions from the respiration signal in daily life. Li showed that brain-derived neurotrophic factor can predict bipolar disorder[10]. However, dedicated equipment is too expensive to analyze brain-derived neurotrophic factor. This study aims to predict future mood in daily life and to find factors of changes in mood. 


\section{HOW TO IMPROVE MENTAL HEALTH}

\section{A. Outline of Mental Care System}

This study aims to develop a mental health system used in the daily life cycle of users. The system is used in the way depicted in figure 1:

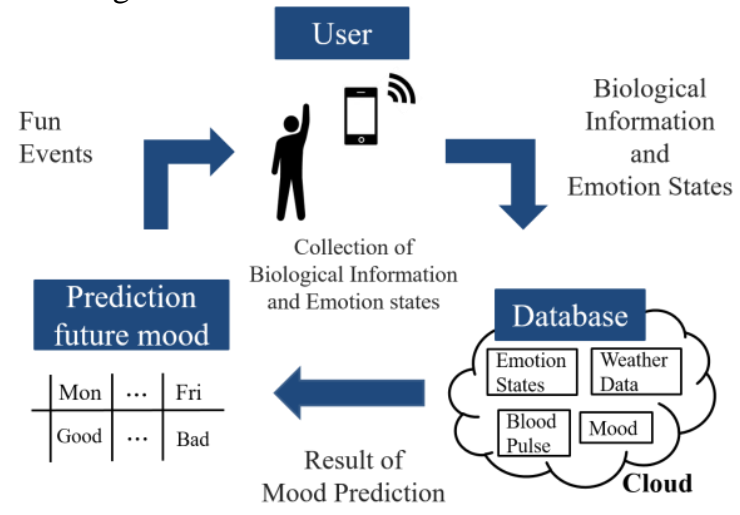

Fig. 1: Mental care system

Biological information is collected by wearable devices every day. The biological information includes the blood pulse, the emotion and the mood. Emotion states are collected through events which inducts emotion. The data are accumulated in an individual database server for long term. The data are used to predict the mood in the future. The system predicts user's future depression instead of the user. If a future depression is detected, the system presents fun events to improve future mood of the user.

\section{B. Prediction and Improvement of Mood}

Extending on the model proposed in the previous study [11], the method this paper presents predicts future mood. It improves future mood with factors effective to change mood. The mood prediction model in [11] needs to be enhanced to be more accurate one, using biological information and weather information. The integration of cognitive emotion to the mood prediction model can improve the accuracy of mood prediction. The method is carried out in the way depicted in figure 2:

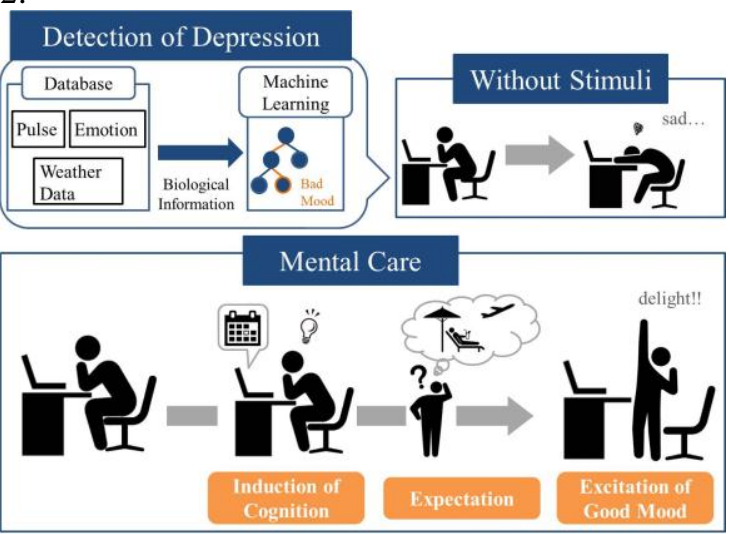

Fig. 2: Proposed method

1. Collection of biological information, weather information, cognitive emotion, and mood,

2. Construction of a mood prediction model using these data,

3. Detection of future depression,
4. Induction of cognition by making the user notice fun events in the future,

5. Improvement of mood through expectation of the future.

\section{How to Collect the Biological Information}

We collect the biological information such as the blood pulse. The blood pulse was collected by a pulse meter worn on the left wrist throughout the day. The blood pulse in the day time represents physiological changes when cognitive emotions happen. The blood pulse in the sleeping time represents mood changes because mood depression causes sleep disorders.

\section{How to Evaluate Emotion and Mood}

Users presented their cognitive emotion to a smart phone using KOKOROSCALE [11], a two-dimensional coordinate systems designed to measure the intuitive emotion easily. KOKOROSCALE is based on Rolls' theory [13] which distinguishes 4 category of emotion. To collect emotion, we use one instance of KOKOROSCALE, where the $X$ axis represents fun or sadness, while the $\mathrm{Y}$ axis represents delight or unwilling. We use another instance of KOKOROSCALE to collect data on mood once a day, where the $\mathrm{X}$ axis represents relief or fear while the $\mathrm{Y}$ axis represents elation or depression. Each of the two-dimensional coordinate systems is shown in fig 3:
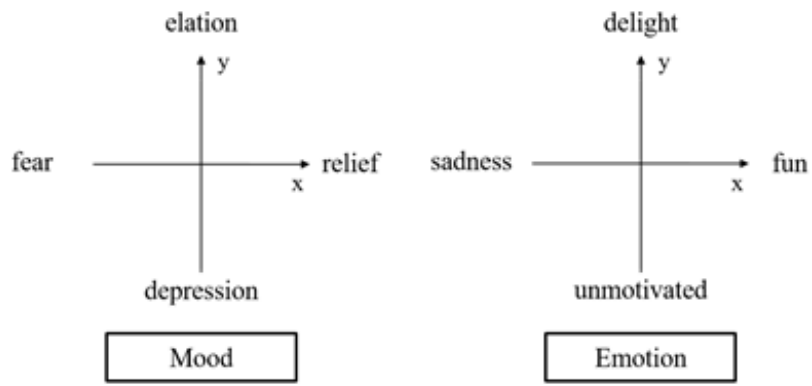

Emotion

Fig. 3: Evaluation of mood and emotion

Users register their schedules until a week later. 3 days or 1 day before an events registered in the schedule, a reminder of the event pops up on users' smart phones. If users get aware of the pop-up, their' cognitive emotion happen. They evaluate their cognitive emotion, using the 2 instances of KOKOROSCALE. Users' mood is evaluated every day in this way.

\section{E. Mood Prediction Model}

We used the random forest to construct a mood prediction model. Random forest is a classification algorithm using an ensemble of tree-structured classifiers. The random forest makes many trees independently constructed using a bootstrap sample of the training data. The majority vote from trees decides the prediction. The advantages of the random forest are bagging with random features. In the bagging, the random forest chooses features at random to reduce correlations among each tree. It suppresses the generalization error of tree classifiers caused by the strength of the trees and the 
correlations between them. The random forest has better accuracy than other algorithms using all features.

\section{EXPERIMENT}

\section{A. Experimental Procedure}

We conducted an experiment on one male and one female for two months in order to investigate the accuracy of the mood prediction and the factors which affect future mood. We collected the blood pulse, the weather information, the current mood, and the cognitive emotion as the factors of the mood prediction. The blood pulse (BP) was collected by the wearable pulse mater, EPSON PS-100BL. We used the temperature (TP) and the length of sunshine duration (LSD) as the weather information. Information on future events includes examinations (E), leisure (LS), part-time jobs (PTJ), and club activities (CA). We used the emotion which is evaluated by an instance of KOKOROSCALE. The $\mathrm{X}$ axis represents fun or sadness $(\mathrm{X})$ and the $\mathrm{Y}$ axis represents delight or unwilling $(\mathrm{Y})$. We collected the mood which is evaluated with another instance of KOKOROSCALE. The $\mathrm{X}$ axis represents relief or fear $(\mathrm{Mx})$, while the $\mathrm{Y}$ axis represents elation or depression (My).

\section{B. Evaluation}

We made two patterns of the mood prediction using RF, i.e., a model with emotion and one without it, in order to compare the prediction accuracy. We used 14 predictor variables. They include the average, the maximum, the minimum, and the standard deviation of BP in awaking, which are denoted with ABP, MaxBP, MinBP, and STBP, respectively. They include $B P$ in sleeping in the same way, denoted as SABP, MaxSBP, MinSBP, and STSBP. The average, the maximum, and the minimum of TP and LSD also predictor variables, which are AT, MaxT, and MinT, respectively. The last predictor variables are the strongest value in the $\mathrm{X}$-axis of the emotion instance of KOKOROSCALE, that is fun or sadness, for any event on the day, and that in the Y-axis, delight or unmotivated. They are denoted as Ex and Ey, respectively. The objective variables correspond to the mode from the next day to the 7 days later, that is, Mx_t and My_t, $(\mathrm{t}=1,2,3 \ldots 7)$. To evaluate the prediction accuracy, we used the F-measure, F, which is the harmonic mean of the precision, $\mathrm{P}$, and the recall, $\mathrm{R}$ :

$$
F=2 * P * R / P+R
$$

We used the leave-one-out cross-validation to investigate the accuracy of the mood prediction. The data set is divided into $\mathrm{K}$ subsets, and the holdout method is repeated $\mathrm{K}$ times $\mathrm{N}$, the number of data points in the set. This means that at $\mathrm{N}$ separate time, the function is trained on all of the data except for one point. The prediction is made for that point. In addition, we investigated the correlations between emotion and future mood to find out what kind of emotion affects mood. Since E and CA are too few to investigated correlations, these features only used for the calculation of Ex and Ey. We also evaluated the contribution to investigate the factors affecting the accuracy of the mood prediction. The contribution is calculated by selection of features which construct the decision trees.

\section{Result}

The prediction results of Mx_t, My_t for the male and the female are shown in Table1 and Table 2, respectively. In the tables, f and f_n are the F-measure with emotion and that without it. As Table 1 shows, the average of $\mathrm{f}$ is higher than the average of $f \_n$ in male. In contrast, Table 2 shows $f$ is less than $\mathrm{f} \_\mathrm{n}$ in female.

TABLE I: F-MEANS PREDICTING MALE's MoOD

\begin{tabular}{llllll}
\hline \hline Mx_t & F & f_n & My_t & f & f_n \\
\hline Mx_1 & 0.310 & 0.286 & My_1 & 0.394 & 0.394 \\
Mx_2 & 0.438 & 0.299 & My_2 & 0.571 & 0.571 \\
Mx_3 & 0.725 & 0.825 & My_3 & 0.643 & 0.560 \\
Mx_4 & 0.379 & 0.419 & My_4 & 0.429 & 0.429 \\
Mx_5 & 0.646 & 0.588 & My_5 & 0.444 & 0.444 \\
Mx_6 & 0.375 & 0.371 & My_6 & 0.444 & 0.444 \\
Mx_7 & 0.293 & 0.348 & My_7 & 0.482 & 0.482 \\
Average & 0.452 & 0.448 & Average & 0.487 & 0.475 \\
\hline \hline
\end{tabular}

TABLE II: F-MEANS PREDICTING FEMALE'S MOOD

\begin{tabular}{llllll}
\hline \hline Mx_t & f & f_n & My_t & f & f_n \\
\hline Mx_1 & 0.411 & 0.458 & My_1 & 0.455 & 0.455 \\
Mx_2 & 0.459 & 0.459 & My_2 & 0.304 & 0.304 \\
Mx_3 & 0.524 & 0.524 & My_3 & 0.391 & 0.391 \\
Mx_4 & 0.227 & 0.227 & My_4 & 0.342 & 0.267 \\
Mx_5 & 0.294 & 0.333 & My_5 & 0.438 & 0.675 \\
Mx_6 & 0.764 & 0.764 & My_6 & 0.409 & 0.409 \\
Mx_7 & 0.333 & 0.333 & My_7 & 0.250 & 0.294 \\
Average & 0.430 & 0.433 & Average & 0.370 & 0.399 \\
\hline \hline
\end{tabular}

Table 3 and 4 show the correlation between the events and the mood of 3 days or 1day before the events. As table 3 shows, correlation between X of LS and My_3 is especially strong in the male. Table 4 shows that the correlation is generally low in the female.

TABLE III: MALE'S CORRELATIONS

\begin{tabular}{lllll}
\hline \hline \multirow{5}{*}{ Event } & Emotion & Date & $\mathrm{Mx}$ & $\mathrm{My}$ \\
\hline PTJ & $\mathrm{x}$ & 3 days & -0.351 & -0.047 \\
& & 1 days & -0.123 & -0.343 \\
& $\mathrm{y}$ & 3 days & -0.516 & -0.273 \\
& & 1 days & -0.352 & -0.652 \\
LS & $\mathrm{x}$ & 3 days & 0.463 & 0.837 \\
& & 1 days & -0.460 & 0.690 \\
& $\mathrm{y}$ & 3 days & -0.071 & 0.333 \\
& & 1 days & 0.683 & 0.742 \\
\hline \hline
\end{tabular}

TABLE IV: FEMALE'S CORRELATIONS

\begin{tabular}{lllll}
\hline \hline Event & Emotion & Date & $\mathrm{Mx}$ & $\mathrm{My}$ \\
\hline PTJ & $\mathrm{x}$ & 3 days & 0.101 & -0.145 \\
& & 1 days & 0.174 & -0.093 \\
& $\mathrm{y}$ & 3 days & -0.002 & -0.338 \\
& & 1 days & 0.312 & -0.237 \\
LS & $\mathrm{x}$ & 3 days & 0.241 & 0.554
\end{tabular}




\begin{tabular}{|c|c|c|c|}
\hline & 1 days & -0.320 & -0.136 \\
\hline \multirow[t]{2}{*}{$\mathrm{y}$} & 3 days & -0.086 & 0.418 \\
\hline & 1 days & 0.034 & 0.386 \\
\hline
\end{tabular}

Table 5 shows contribution of mood prediction for the first three places when $\mathrm{f}$ is higher than $50 \%$.

TABLE V: THE FIRST THREE PLACE OF CONTRIBUTION

\begin{tabular}{lllll}
\hline \hline Gender & Mood & First & Second & third \\
\hline Male & Mx_3 & SABP & SBP(min) & Ex \\
& Mx_5 & Ex & IS & TP(min) \\
& My_2 & Ey & STBP & ABP \\
& My_3 & IS & Ex & BP(max) \\
Female & Mx_3 & ABP & IS & STBP \\
& Mx_6 & ATP & SABP & Ey \\
& My_5 & TP(max) & STBP & TP(min) \\
\hline \hline
\end{tabular}

D. Discussion

According to Table 1 and 3, cognitive emotion is effective in case of predicting the male's mood. In fact, Table 5 shows that the contribution of emotion is generally high. Especially, $f$ predicting My_3 is the highest. The accuracy of My_3 is the best because the correlation between My_3 and X of LS is 0.837 . This shows that positive emotion strongly relates to future elation. In contrast, cognitive emotion is not effective to predict female's mood. As Table 2 and Table 4 show, correlation does not strongly appear between mood and cognitive emotion, and the contribution of cognitive emotion is generally low. Therefore, in case of female, cognitive emotion for E, LS, PTJ, and, CA is not important events to affect mood. This result means difference of character and gender is affecting mood prediction, because of difference of cognitive for events. IS and STBP have high contribution in common between male and female. It shows that amounts of changing in the blood pulse and whether sunny or not. Previous study [11] shows that the samples for mood prediction need the amount of four months' data at minimum. However, in this study, there is the amount of three month's data. It seems to be a reason why the prediction accuracy is scattering.

\section{E. Conclusion}

The paper proposes a prediction model of the future mood using data collected from users. The average of classifying elation or depression using cognitive emotion improved the prediction accuracy by $1.2 \%$ in case of male. Furthermore, providing individuals with events that induce their fun expectations can improve their future mood. However, in case of female, the average of the prediction accuracy using emotion is lower than that without emotion. The cause for difference of the prediction accuracy is probably considered that difference of character and gender are affecting. We need further experiences considering individual characters and gender. We will also collect more sample data in order to reduce the scatter of the prediction accuracy. A mood prediction model has possibility to contribute to the self-care of mental health conditions. We can help users avoid future depression while encouraging them to expect fun.

\section{REFERENCES}

[1] 10 FACTS ON MENTAL HEALTH - Mental health: a state of wellbeing (Report). WHO. (2014-08).

[2] Eagle, Charles Thomas. "Effects of existing mood and order of presentation of vocal and instrumental music on rated mood responses to that music." Bulletin of the Council for Research in Music Education, No.32, pp.55-59 (1973)

[3] Frijida, N.H." The law of emotion, American Psychologist," Vol.43 (1988), pp.349-358 http://dx.doi.org/10.1037/0003-066X.43.5.349

[4] Sloboda, John A., and Patrik N. Juslin. "At the interface between the inner and outer world." Handbook of music and emotion (2010): 73-97.

[5] Beedie, Christopher, Peter Terry, and Andrew Lane. "Distinctions between emotion and mood." Cognition \& Emotion 19.6 (2005): 847 878

http://dx.doi.org/10.1080/02699930541000057

[6] Cannon, Walter B. "The James-Lange theory of emotions: A critical examination and an alternative theory." The American Journal of Psychology (1927): 106-124. http://dx.doi.org/10.2307/1415404

[7] Lazarus, Richard S. "Psychological stress and the coping process." (1966).

[8] Katsimerou, C., Heynderickx, I., \& Redi, J. Predicting mood from punctual emotion annotations on videos.

[9] Wu, Chi-Keng, Pau-Choo Chung, and Chi-Jen Wang. Representative segment-based emotion analysis and classification with automatic respiration signal segmentation. Affective Computing, IEEE Transactions on 3.4 (2012): 482-495.

[10] $\mathrm{Li}$, Zezhi, et al. "Brain-derived neurotrophic factor levels and bipolar disorder in patients in their first depressive episode: 3-year prospective longitudinal study." The British Journal of Psychiatry 205.1 (2014): 2935 . http://dx.doi.org/10.1192/bjp.bp.113.134064

[11] Kajiwara et al. "A Mood Prediction System for Preventing Diseases Using Biological Information and Weather Information" IEEJ Transactions on Electronics on 135.6 (2015): 570-579. (in Japanese) http://dx.doi.org/10.1541/ieejeiss.135.570

[12] http://www.riken.jp/pr/press/2012/20120301/ (in Japanese)

[13] E.T.Rolls : A theory of emotion, and its application to understanding the neural basis of emotion, Neural and Chemical Control, pp.325344, Japan Scientific Societies Press, 1986 